\title{
Comparison of Keratometry, Central Corneal Thickness, and Anterior Chamber Depth Results Measured With Nidek-AL Scan Biometry and Sirius Topography Devices
}

\author{
Sadık Etka Bayramoğlu (1), Nihat Sayın (1D, Dilbade Yıldız Ekinci (1), Mehmet Erdoğan (1)
}

Introduction: To investigate whether the central corneal thickness (CCT), anterior chamber depth (ACD), and keratometric measurements change according to biometric and topographic measurements in childhood and in young adult age.

Methods: In total, 147 eyes of 78 patients were included in the study. Patients with an ocular disease other than refractive error were not included in the study. CCT, ACD, and keratometry values of all patients were measured with Nidek-AL Scan optical biometry and Sirius combined Scheimpflug-Plasido disc Corneal Topography devices.

Results: Of the seventy-eight patients, 46 were female and 32 were male. The mean age was $14.46 \pm 5.15$ years (range, $5-29$ years), the mean spherical equivalence was $-0.30 \pm 1.13$ diopters (D), and the mean axial length was $23.28 \pm 0.77 \mathrm{~mm}$. The Sirius and AL-Scan CCT were $555.66 \pm 39.58$ $\mu \mathrm{m}$ and $548.01 \pm 38.14 \mu \mathrm{m}$, respectively. The Sirius and AL-Scan ACD were $3.65 \pm 0.28 \mathrm{~mm}$ and $3.57 \pm 0.26 \mathrm{~mm} \mu \mathrm{m}$, respectively. The Sirius SimK, AL-Scan K $2.4 \mathrm{~mm}$, and AL-Scan K $3.3 \mathrm{~mm}$ values were 43.09 $\pm 1.45 \mathrm{D}, 43.22 \pm 1.50 \mathrm{D}$, and $43.2 \pm 1.47 \mathrm{D}$, respectively. The values of CCT and ACD measured on the Sirius device were found to be statistically significantly higher than those measured by the AL-Scan device $(p=.00)$. A high level of correlation was found between the Sirius CCT and the AL-Scan CCT $(p=0.000, r=0.974)$. A high level of correlation was found between the Sirius ACD and AL-Scan ACD ( $p=0.000, r=0.918)$. A high level of correlation was found between the the Sirius SimK value and the AL-Scan K 2.4 $\mathrm{mm}$ and $\mathrm{K} 3.3 \mathrm{~mm}$ values ( $\mathrm{p}=0.000$, $r$ values were 0.979 and 0.982 , respectively).

Conclusion: In childhood and young adult age, between the Sirius and AL-Scan devices, although CCT, ACD, SimK, and 2.4-3.3 mm K were statistically different, a high correlation and agreement were found between the measurements.

Keywords: Biometry, corneal topography, pachymetry, sirius, AL-Scan

This study was presented at $1^{\text {st }}$ Live Surgery Symposium, 16-18 June 2017, İstanbul, Turkey.

ORCID IDs of all authors: S.E.B. 0000-0002-95024368; N.S. 0000-0002-1442-9743; D.Y.E. 00000002-5535-264X, M.E. 0000-0002-2556-7383.

Clinic of Ophthalmology, Health Sciences University Kanuni Sultan Süleyman Training and Research Hospital, İstanbul, Turkey

Address for Correspondence: Sadık Etka Bayramoğlu E-mail: sadiketka@windowslive.com

Received: 30.09 .2017 Accepted: 17.12.2017

(C) Copyright 2018 by Available online at istanbulmedicaljournal.org

\section{Introduction}

In addition to biomicroscopic examination, the use of scanner slit topography, interferometry, Scheimpflug imaging, and optical coherence tomography devices is increasing for preoperative evaluation or patient follow-up to diagnose the anterior segment. More accurate measurements of the anterior segment parameters through imaging methods reduce diagnostic and treatment errors. The technical characteristics of the devices and the measurement techniques influence the repeatability and comparability of the measurements of patient characteristics.

Nidek AL-Scan (Nidek, Aichi, Japan) is an optical biometry device used to calculate the intraocular lens $(\mathrm{IOL})$ degree before cataract surgery. The main advantages of the device are minimizing the user-induced measurement errors and the errors resulting from eye movement because it is a 3D automatic eye movement monitoring system and the absence of measurement errors caused by corneal indentation due to the fact that it is a non-contact measuring device (1).

The Sirius topographer (Costruzione Strumenti Ophthalmologist, Florence, Italy) is a frontal segment analyzer that evaluates all the data obtained from a rotating Scheimpflug camera and a Placido disc imaging system. Topographic measurements that include anterior-posterior corneal elevation maps, corneal thickness, anterior chamber depth (ACD), and keratometry are the basic measurement parameters (2). The diagnosis and follow-up of corneal diseases are used for evaluation before refractive surgery and for its follow-up process.

Effective lens position (ELP) is the measured distance between the cornea and the IOL after cataract surgery (3). An incorrect estimate of the ELP position is the most important cause of refractive errors after cataract surgery (4). The first and second generation lens power calculation formulas calculate ELP using the axial length and keratometry values (5). New generation formulas, such as Holladay 2 and Haigis, use ACD in ELP calculation $(3,5,6)$. Errors in the measurement of ACD result in the miscalculations of ELP and postoperative refractive errors in these formulas (6).

The aim of the present study was to determine whether there is a difference in central corneal thickness (CCT) and ACD parameters in measurements obtained from Nidek AL-Scan and Siri- 
us topography devices, which are reported to measure CCT and ACD parameters in childhood and young adulthood by using the Scheimpflug method with high repeatability $(2,7)$.

\section{Methods}

One hundred forty-seven eyes of 78 patients who applied to an outpatient clinic examination of Kanuni Sultan Süleyman Training and Research Hospital Clinic of Ophthalmology were included in the study. The study was conducted in accordance with the principles of the Declaration of Helsinki. Parental or guardian consent was obtained from the legal parents or guardians of all patients.

A thorough ophthalmologic examination that included refraction, biomicroscopy, and ophthalmic examination was performed on all patients. Patients with additional ocular diseases other than refractive error and who underwent intraocular surgery were excluded from the study. Patients with $>5$ diopters (D) of myopia and $>3 \mathrm{D}$ of hyperopia were also excluded.

\section{Measuring devices}

The Sirius topography instrument is an anterior segment analysis system combining a monochromatic 360-degree rotating Scheimpflug camera with a 22-ring Placido disc. It takes 25 radial sections from the cornea and the front chamber. It calculates the corneal anterior surface measurements by analyzing the software of Scheimpflug camera and Placido disc measurements. All other measurements for internal structures are derived from the Scheimpflug camera.

The Nidek AL-Scan instrument measures ACD and CCT with the aid of Scheimpflug imaging and performs AL measurements via partially coherent laser interferometry technology (7). The corneal refractive power and the flattest and steepest meridians of the cornea are calculated using a photodetector by calculating the rings projected onto the patient's cornea.

\section{Measurement technique}

All measurements were performed by the same doctor from 12:00 pm to 13:30 pm on Sirius and AL-Scan. Each measurement had at least 5-minute intervals. Measurements were obtained on the ALScan and Sirius devices, and patients were instructed to blink their eyes after each internal fixation light shot. The measurement was recorded after an "OK" report was received on the Sirius device, indicating that the measurement was of sufficient quality. During the AL-Scan device measurement when it was determined that the patient did not blink and had not fixated their eyes on the light, the procedure was repeated until sufficient measurement was obtained.

Measurements for ACD, flat K, steep K, mean SimK, CCT, iris diameter, iridocorneal angle, and anterior chamber volume (ACV) were recorded by the Sirius topographer. The value expected to be measured is obtained using Javal keratometry, and the SimK value is obtained after the Sirius instrument calculation. The width of the calculated zone varies according to the curvature of the measured cornea. CCT, ACD, $2.4 \mathrm{~mm} \mathrm{~K}$ flat, $2.4 \mathrm{~mm} \mathrm{~K}$ steep, $2.4 \mathrm{~mm} \mathrm{~K}$ average, $3.3 \mathrm{~mm} \mathrm{~K}$ flat, $3.3 \mathrm{~mm} \mathrm{~K}$ steep, $3.3 \mathrm{~mm} \mathrm{~K}$ average, and axial length were measured by the Nidek AL-Scan device.

\section{Statistical Analysis}

Statistical Package for Social Sciences software version 18.0 for Windows (SPSS Inc.; Chicago, IL, USA) was used for statistical analysis. The Kolmogorov-Smirnov test was used to assess whether the data fit the normal distribution. The CCT, ACD, flat K-steep K-Sim K average, flat K 2.4 mm-steep K 2.4 mm-2.4 mm K, and 3.3 mm flat K-3.3 $\mathrm{mm}$ steep K-3.3 mm K values corresponding to the normal distribution were compared with paired t test. The CCT, ACD, and SimK values and the $2.4 \mathrm{~mm} \mathrm{~K}-3.3 \mathrm{~mm} \mathrm{~K}$ values that were measured in both devices were assessed in absolute agreement with Pearson's correlation analysis and intraclass correlation coefficient (ICC).

\section{Results}

Of the 78 patients, 46 were females, and 32 were males. The average age of the patients was $14.46 \pm 5.15$ (between 5 and 29) years, and the mean spherical equivalence was $-0.30 \pm 1.13$ D. Table 1 shows the basic ocular measurement parameters obtained from the Sirius topography and AL-Scan devices.

Sirius CCT was $555.66 \pm 39.58 \mu \mathrm{m}$, and AL-Scan CCT was $548.01 \pm 38.14 \mu \mathrm{m}$. Sirius ACD was $3.65 \pm 0.28 \mathrm{~mm}$, and AL-Scan ACD was $3.57 \pm 0.26 \mathrm{~mm}$. The Sirius CCT and Sirius ACD values were found to be statistically significantly higher than the AL-Scan values. In keratometry measurements, the Sirius SimK values were statistically significantly lower than the $2.4 \mathrm{~mm} \mathrm{AL-Scan}$ and 3.3 $\mathrm{mm}$ AL-Scan values. Table 2 shows the mean difference for all measurements between both devices, the $95 \%$ confidence interval for the difference, and the $p$-value.

A high level of correlation was found between Sirius CCT and AL-Scan CCT ( $p=0.000, r=0.974)$. A high level of correlation was found between Sirius ACD and AL-Scan ACD ( $p=0.000, r=0.918)$. A high correlation was found between the Sirius SimK value and the AL-Scan $\mathrm{K} 2.4 \mathrm{~mm}$ and $\mathrm{K} 3.3 \mathrm{~mm}$ values $(\mathrm{p}=0.000$, $\mathrm{rs}=0.979$ and 0.982 , respectively) (Table 3 ).

The ICC evaluated for absolute agreement between the Sirius CCT and the AL-Scan CCT tests shows high agreement. A high level of agreement was found between Sirius ACD and AL-Scan ACD. A high

\begin{tabular}{lccc}
\multicolumn{4}{c}{ Table 1. Average measurements for both devices } \\
\cline { 3 - 4 } & Measuring device & Average & SD \\
CCT & Sirius & $555.66 \mu \mathrm{m}$ & 39.58 \\
ACD & Sirius & $3.65 \mathrm{~mm}$ & 0.26 \\
\hline SimK & Sirius & 43.09 & 1.45 \\
\hline Iris diameter & Sirius & $12.33 \mathrm{~mm}$ & 0.38 \\
\hline ACV & Sirius & 163.55 & 25.13 \\
ICA & Sirius & 43.83 & 5.75 \\
\hline CCT & AL-Scan & $548.01 \mu \mathrm{m}$ & 38.14 \\
ACD & AL-Scan & $3.57 \mathrm{~mm}$ & 0.26 \\
K 2.4 mm & AL-Scan & 43.22 & 1.5 \\
K 3.3 mm & AL-Scan & 43.2 & 1.47 \\
Axial length & AL-Scan & $23.3 \mathrm{~mm}$ & 0.75
\end{tabular}

CCT: central corneal thickness, ACD: anterior chamber depth, K: keratometry, ACV: anterior chamber volume, ICA: iridocorneal angle; SD: standart deviation 
Table 2. Comparison of CCT, ACD, and keratometry data obtained from Sirius and AL-Scan

\begin{tabular}{|c|c|c|c|c|c|}
\hline & & & $95 \%$ confide & of difference & \\
\hline & & Average difference & Lower limit & Upper limit & $\mathbf{p}^{*}$ \\
\hline CCT Sirius & CCT AL-Scan & 7.56 & 6.08 & 9.03 & 0.000 \\
\hline ACD Sirius & ACD AL-Scan & 0.07 & 0.06 & 0.09 & 0.000 \\
\hline Sirius SimK & $\mathrm{K} 2.4 \mathrm{~mm}$ AL-Scan & -0.102 & -0.152 & -0.051 & 0.000 \\
\hline Sirius SimK & K 3.3mm AL-Scan & -0.090 & -0.136 & -0.045 & 0.000 \\
\hline K flat Sirius & $\mathrm{K}$ flat $2.4 \mathrm{~mm}$ AL-Scan & -0.063 & -0.108 & -0.019 & 0.006 \\
\hline $\mathrm{K}$ flat Sirius & $\mathrm{K}$ flat $3.3 \mathrm{~mm}$ AL-Scan & -0.041 & -0.081 & 0.000 & 0.047 \\
\hline K steep Sirius & $\mathrm{K}$ steep $2.4 \mathrm{~mm}$ AL-Scan & -0.136 & -0.201 & -0.071 & 0.000 \\
\hline K steep Sirius & $\mathrm{K}$ dik 3.3mm AL-Scan & -0.107 & -0.172 & -0.041 & 0.002 \\
\hline $\mathrm{K} 2.4 \mathrm{~mm}$ AL-Scan & K 3.3mm AL-Scan & 0.014 & -0.009 & 0.037 & 0.225 \\
\hline $\mathrm{K}$ flat $2.4 \mathrm{~mm} \mathrm{AL-Scan}$ & $\mathrm{K}$ flat $3.3 \mathrm{~mm} \mathrm{AL-Scan}$ & 0.023 & 0.001 & 0.044 & 0.037 \\
\hline K steep $2.4 \mathrm{~mm}$ AL-Scan & K steep $3.3 \mathrm{~mm}$ AL-Scan & 0.029 & -0.010 & 0.069 & 0.146 \\
\hline
\end{tabular}

Table 3. Pearson correlation analysis and absolute agreement analysis with ICC

\begin{tabular}{llccccc} 
& & $\begin{array}{c}\text { Pearson correlation } \\
\text { coefficient }\end{array}$ & ICC & Lower limit & Upper limit & p confidence interval according to ICC \\
\hline CCT Sirius & CTT AL-Scan & 0.974 & 0.977 & 0.89 & 0.991 & 0.00 \\
\hline ACD Sirius & ACD AL-Scan & 0.918 & 0.935 & 0.806 & 0.969 & 0.00 \\
\hline Sirius SimK & K 2.4mm AL-Scan & 0.979 & 0.988 & 0.982 & 0.992 & 0.00 \\
\hline Sirius SimK & K 3.3mm AL-Scan & 0.982 & 0.99 & 0.985 & 0.993 & 0.00 \\
\hline \multicolumn{2}{l}{ CCT: central corneal thickness, ACD: anterior chamber depth, K: keratometry } & & & &
\end{tabular}

level of agreement was found between the Sirius SimK value and the AL-Scan K $2.4 \mathrm{~mm}$ and $\mathrm{K} 3.3 \mathrm{~mm}$ values. Table 3 shows the ICC between the measurements and the $95 \%$ confidence interval values according to the ICC.

\section{Discussion}

Many factors affect postoperative refraction, but lens power calculation formulas that are used, axial length, postoperative ELP, and corneal curvature are the main factors $(3,8,9)$. The preoperative ACD value is the main determinant value in the formula developed by Olsen in calculating postoperative ELP (6). For this reason, ACD has critical importance for refractive outcomes after cataract surgery.

A decreased ACD increases the risk of intraoperative complications in intraocular surgeries. In patients with pseudoexfoliation syndrome, zonal instability increases in the eyes with an ACD of $<2.5$ $\mathrm{mm}$ (10). ACD is an important parameter for evaluating the risk of angle closure and endothelial cell injury in iris-fixated phakic IOL implantation $(11,12)$.

Nidek AL-Scan is a biometry device produced for the accurate calculation of the IOL power before cataract surgery. The most common use is to make measurements and calculations for the best refractive result for IOL to be placed. The reliability of the Nidek AL-Scan device for IOL power calculation has been confirmed by previous studies $(13,14)$. It has been shown that the Nidek AL-Scan measurements are repeatable (15).
The Sirius topography device is used for diagnostic evaluation of corneal diseases and preoperative evaluation before refractive surgery and cataract surgery. It has been shown in different studies that the reproducibility and reliability of CCT, ACD, and SimK measurement parameters are very high with the Sirius device $(2,16)$. In a study conducted by utilizing the axial length values that were obtained with keratometry values and immersion ultrasound, which were determined by the Sirius topography device in the 3rd generation lens power calculation formulas, the mean deviation of postoperative refractive was found to be $0.23 \pm 0.24 \mathrm{D}$ (17). It has been reported that good results can be obtained using the Sirius topography device in the eyes that have not been operated previously (17).

Front segment topography devices and biometry devices were compared in different studies. In a study comparing the ACD in the Galilei device and Al-Scan devices that use double rotating Scheimpflug camera and Placido topography, despite the higher values obtained in the ACD Galilean device, a high agreement was determined between the measurements (18). It has been suggested in the study that the effect of CCT on the ACD value should be investigated in order to be able to fully understand how much difference of ACD is related to corneal thickness. Yağcl et al. (15) compared Nidek Al-Scan and Galilei devices among normal individuals and patients with keratoconus, and the CCT and ACD values are found to be higher in the Galilei device. In addition, although the $C C T, A C D$, and keratometry values in both devices were found to be highly compatible in normal individuals, in patients with 
keratoconus, only the ACD values were found to have compatible measurements.

In the literature, similar to our study, in a comparative study of $\mathrm{Ni}$ dek AL-Scan and Sirius tomography measurements that were performed on an adult patient group, a high correlation between CCT, ACD, and keratometry measurements was found (19). In our study, a high correlation between CCT, ACD, SimK Sirius, and K 2.4-3.3 mm AL-Scan supports that the correlation continues in all age groups.

In studies comparing Galilei with AL-Scan and Sirius with AL-Scan measurements in normal subjects, the CCT and ACD values that were measured by Galilei and Sirius devices being higher but compatible might result from Galilei and Sirius devices having similar mechanisms.

A correct calculation of CCT is vitally important in pre-refractive surgeries and $\mathrm{IOL}$ calculations in patients with glaucoma. The thicker CCT value that we determined on our Sirius device in our study is a condition that should be considered when evaluating a patient with glaucoma.

\section{Conclusion}

The fact that the biometric ACD was lower in our study than the topographic ACD indicates that the formulas for lens power calculation may give different results depending on the measurements obtained on different devices due to the different measurements between the devices. Although there is a high correlation and agreement between the measurements obtained from both devices, the clinical significance of the difference can be determined by clinical trials by investigating how many postoperative refractive deviations from both devices can be attributed.

Ethics Committee Approval: The ethics committee approval has been received by the Ethics Committee of Sağlık Bilimleri Üniversitesi Kanuni Sultan Süleyman Training and Research Hospital (Approval Number: KAEK/2018.3.3).

Informed Consent: Informed consent was obtained from parents of the patients and patients who participated in this study.

Peer-review: Externally peer-reviewed.

Author Contributions: Concept - S.E.B.; Design - S.E.B.; Supervision - N.S., D.Y.E., M.E.; Resource - S.E.B.; Materials - N.S., D.Y.E., M.E., S.E.B.; Data Collection and/or Processing - S.E.B.; Analysis and/or Interpretation - S.E.B.; Literature Search - S.E.B.; Writing - S.E.B.; Critical Reviews - N.S., D.Y.E., M.E.

Conflict of Interest: The authors have no conflicts of interest to declare.

Financial Disclosure: The authors declared that this study has received no financial support.

\section{References}

1. Kola M, Duran H, Turk A, Mollamehmetoglu S, Kalkisim A, Erdöl H. Evaluation of the repeatability and the reproducibility of AL-scan measurements obtained by residents. J Ophthalmol. 2014. [CrossRef]

2. Savini G, Barboni P, Carbonelli M, Hoffer KJ. Repeatability of automatic measurements by a new Scheimpflug camera combined with Placido topography. J Cataract Refract Surg. 2011; 37: 1809-16. [CrossRef]

3. Olsen T. Prediction of the effective postoperative (intraocular lens) anterior chamber depth. J Cataract Refract Surg 2006; 32: 419-24. [CrossRef]

4. Olsen T. Sources of error in intraocular lens power calculation. J Cataract Refract Surg 1992; 18: 125-9. [CrossRef]

5. Retzlaff JA, Sanders DR, Kraff MC. Development of the SRK/T intraocular lens implant power calculation formula. J Cataract Refract Surg. 1990; 16: 333-40. [CrossRef]

6. Olsen T. Calculation of intraocular lens power: a review. Acta Ophthalmol Scand 2007; 85: 472-85. [CrossRef]

7. Yağcı R, Güler E, Kulak AE, Erdoğan BD, Balcı M, Hepșen IF. Repeatability and reproducibility of a new optical biometer in normal and keratoconic eyes. J Cataract Refract Surg. 2015; 41: 171-7. [CrossRef]

8. Hosny M, Alió JL, Claramonte P, Attia WH, Pérez-Santonja JJ. Relationship between anterior chamber depth, refractive state, corneal diameter, and axial length. J Refract Surg 2000; 16: 336-40.

9. Holladay JT, Musgrove KH, Prager TC, Lewis JW, Chandler TY, Ruiz RS. A three-part system for refining intraocular lens power calculations. J Cataract Refract Surg 1988; 14: 17-24. [CrossRef]

10. Küchle M, Viestenz A, Martus $P$, Händel A, Jünemann A, Naumann GO. Anterior chamber depth and complications during cataract surgery in eyes with pseudoexfoliation syndrome. Am J Ophthalmol. 2000; 129: 281-5. [CrossRef]

11. Saxena R, Boekhoorn SS, Mulder PG, Noordzij B, van Rij G, Luyten GP. Long-term follow-up of endothelial cell change after Artisan phakic intraocular lens implantation. Ophthalmology 2008; 115: 608-13. [CrossRef]

12. Devereux JG, Foster PJ, Baasanhu J, Uranchimeg D, Lee PS, Erdenbeleig $\mathrm{T}$, et al. Anterior chamber depth measurement as a screening tool for primary angle-closure glaucoma in an East Asian population. Arch Ophthalmol 2000; 118: 257-63. [CrossRef]

13. Kaswin G, Rousseau A, Mgarrech M, Barreau E, Labetoulle M. Biometry and intraocular lens power calculation results with a new optical biometry device: comparison with the gold standard. J Cataract Refract Surg. 2014; 40: 593-600. [CrossRef]

14. Suto C, Shimamura E, Watanabe I. Comparison of 2 optical biometers and evaluation of the Camellin-Calossi intraocular lens formula for normal cataractous eyes. J Cataract Refract Surg 2015; 41: 2366-72. [CrossRef]

15. Yağcı R, Kulak AE, Güler E, Tenlik A, Gürağaç FB, Hepşen IF. Comparison of anterior segment measurements with a dual Scheimpflug Placido corneal topographer and a new partial coherence interferometer in keratoconic eyes. Cornea 2015; 34: 1012-8. [CrossRef]

16. Masoud M, Livny E, Bahar I. Repeatability and intrasession reproducibility obtained by the Sirius anterior segment analysis system. Eye contact lens. 2015; 41: 107-10. [CrossRef]

17. Savini G, Barboni P, Carbonelli M, Hoffer KJ. Accuracy of corneal power measurements by a new Scheimpflug camera combined with Placido-disk corneal topography for intraocular lens power calculation in unoperated eyes. J Cataract Refract Surg 2012; 38: 787-92. [CrossRef]

18. Dervişoğulları MS, Totan Y, Gürağaç B. Comparison of anterior chamber depth measurements of Nidek AL-Scan and Galilei Dual Scheimpflug Analyzer. Cont Lens Anterior Eye 2015; 38: 85-8. [CrossRef]

19. Çăglar Ç, Kocamış Si, Demir E, Durmuş M. Comparison of the measurements of a novel optical biometry: Nidek AL-Scan with Sirius and a ultrasound biometry. Int Ophthalmol 2017; 37: 491-8. [CrossRef]

Cite this article as: Bayramoğlu SE, Sayın N, Ekinci DY, Erdoğan M. Comparison of Keratometry, Central Corneal Thickness, and Anterior Chamber Depth Results Measured With Nidek-AL Scan Biometry and Sirius Topography Devices. İstanbul Med J 2018; 19: 158-61. 